\title{
Inferring and Enforcing Geometrical Constraints on a 3D Model for Building Reconstruction
}

\author{
Franck Taillandier \\ Institut Géographique National 2-4 avenue Pasteur, 94160 Saint-Mandé, France \\ franck.taillandier@ign.fr
}

\begin{abstract}
We present a method for inferring and enforcing geometrical constraints on an approximate 3D model for building reconstruction applications. Compared to previous works, this approach requires no user intervention for constraints definition, is inherently compliant with the detected constraints and handles over-constrained configurations. An iterative minimization is applied to search for the model subject to geometric constraints that minimizes the distance to the initial approximate model. Results on real data show the gain obtained by this algorithm.
\end{abstract}

\section{Introduction}

\subsection{Motivations}

Reconstruction of man-made objects or environments from multiple images is of primary importance in many fields such as Computer Aided Design, architectural design, building reconstruction. Taking into account some geometrical properties can greatly improve the quality of reconstruction [1], is essential to reduce the degree of freedom of the problem and to provide a good modelling of the environment.

The application described in this article is part of a global project [2] aiming at automatic building reconstruction using plane primitives as base primitives and a modelling of building as polyhedral models without overhang. Integration of constraints in this scheme enables to reduce the combinatory while taking into account the specificity of man-made environment. The part described here focuses on building reconstruction once topology is known by integrating constraints directly inferred on the model.

\subsection{Related Work}

In building reconstruction applications and more generally in architectural modelling, methods used to reconstruct man-made environments can be classified as "model-based" and "constraints-based". In the former ones, objects are decomposed into known base primitives (parallelepiped, rectangles...) grouped together to build more complex shapes [3, 4, 5, 6, 7, 8]. To identify or to validate building parts, matching is performed between a model and 3D or 2D primitives through reprojection of hypotheses on image gradients or corners [4, 5], perceptual or model-based grouping and model verification [6, 7, 8]). Constraints are a priori known in the model and must be enforced on 
the matched primitives for reconstruction purposes, which is a difficult task because redundant constraints and over-constraints are not handled in the geometrical definition of the model. The latter methods are more general. They only rely on some geometrical properties very common in urban or man-made environments, without a priori models, to reconstruct any polyhedral shape. Several works take advantage of these properties [1, 9, 10, 11, 12], but due to the complexity of the problem, they all rely on user intervention for topological and geometrical description of models, which is very time-consuming and often redhibitory for dense and large urban areas reconstruction.

As for the techniques used to enforce constraints on an approximate 3D model, geometrical conditions are expressed in two different ways. Some embed the conditions in the Least Square minimization process [11, 12,1] thus leading to a solution where it is hard to evaluate whether constraints are actually verified or not. Other perform an implicit modelling of the scene before the minimization process [10, 9]. It has the advantage of inherently being compliant with the provided constraints. However, for these systems, although methods are available to detect over-constrained situations, no automatic solution is provided.

As for the constraints handled by the authors, all of them use planarity information to enforce topology constraints that intrinsically result from object description (points belong to the same facet and thus to the same plane). [10,9] use also purely geometrical constraints that arise between object features: orthogonality, parallelism...

\subsection{General Scheme}

The algorithm presented in this article extends the model developed in [9] to avoid the drawbacks recalled in section 1.2. First, constraints are inferred automatically on the initial shape. No user intervention is needed. Then constraints are enforced on the model and degeneracies or over-constraints are handled by iteratively suppressing some constraints. The application presented is dedicated to automatic building reconstruction therefore we focus on constraints recurrent in these environment: parallelisms, orthogonality, horizontal edges, vertical symmetry.

The algorithm assumes an initial approximate 3D model whose topological relations are known. In our example, this model may come from automatic algorithms [2]. In a first step, normal vectors of the different facets are clustered (section 2.2), enabling to handle parallelism properties and horizontal planes. A constraints graph is then deduced from these clusters, integrating all the geometrical constraints that will be applied on the model: orthogonality, horizontal edges, symmetries (sections 2.3 and 2.4). In a second step, a set of equations is built from planarity equations between points and normal vectors. This implicit parameterization ensures that all constraints are verified (section 3.1). Through iterative minimization, one searches then for the constrained model that minimizes the distance to the initial approximate model (section 3.2). Over-constraints situations are detected and handled by iteratively suppressing some constraints until convergence (section 4) A qualitative and quantitative evaluation assess the validity of the approach controlled by two parameters $\sigma_{/ /}$and $\sigma_{\varangle}$ that are easily choosen according to the noise present in the approximate model (section 5). 


\section{Inferring Constraints}

\subsection{Topology and Geometry: Notations}

The application depicted here aims at building roof geometrical reconstruction. Facades making up the border of buildings are inferred from the roofs definition. They will be of primary importance to impose constraints on the external edges of buildings. A facet is added to the initial set of facets for each external edge of the building, therefore what will be called a facet is not necessarily a closed polygon but may be constituted of only two points.

In the following, for the initial model whose topological relations are known, $\mathcal{F}$ is the set of facets whose cardinal is $F$. From the topological point of view, each facet $f$ has $|f|$ points and from a geometrical point of view, it is linked to a normal vector or direction $\mathbf{v}_{i}$. The set of directions $\mathcal{D}$ has cardinality $D$ (initially $D=F$ ). Figure 1(a) shows an example of a 3D building and its schematic representation.

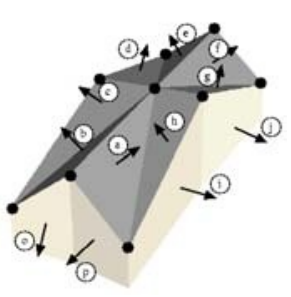

(a)

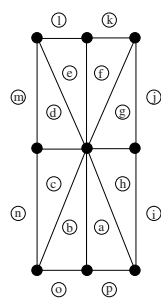

(b)

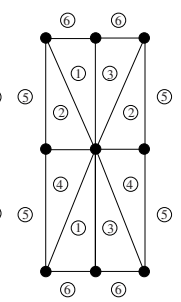

(c)

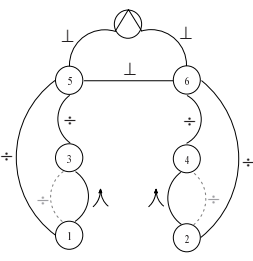

(d)

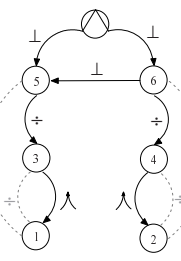

(e)

Fig. 1. Approximate 3D building (a) and its schematic representation with directions (letters) annotated on facets (b) where black circles are initial approximate points that do not verify constraints and vertical planes making up the border are inferred from them. (c) shows the result of clustering. (d) and (e) depict constraints graph and dependences graph respectively. Each arc represents a constraint between two directions. $\oplus$ defines the vertical direction, $\perp, \div$ and $入$ symbolize orthogonality, horizontal edge and vertical symmetry constraints respectively. Grey arcs are constraints suppressed from the graph (see text for details). The sequence of directions of this dependences graph is $(\oplus, 5,4,2,6,3,1)$ and renumbering can be performed

\subsection{Directions Clustering}

From the initial model, all normal vectors are estimated for each facet of the model, thus leading to $F$ normal vectors hereafter called directions $\mathbf{v}$. To these directions, in the case of building reconstruction, the vertical normal is added because of its obvious importance in man-made scene. In order to handle parallelism and horizontal properties, one has to identify directions nearly identical and directions close to the vertical direction. To achieve this, a pairwise centroid-linkage clustering is performed on the initial directions. A cluster is created by grouping the couple of normal vectors whose distance is the lowest. In this case, the distance is the absolute value of the angle between both 
normal vectors. A new normal is recomputed for the new cluster by the mean value of the normals enclosed by the cluster. Iteratively, normals are grouped together and the process goes on until the minimum distance is above a threshold $\sigma_{/ /}$. At the end of the process, each pair of clusters are distant of more than $\sigma_{/ /}$and there is only $D \leq F$ directions. Let us mention that, in the case of building reconstruction applications, in order not to alter the "vertical" direction, the value of the direction for its cluster is kept vertical and not averaged, thus enabling to handle facets considered as horizontal.

Each facet of the initial model is then identified with a direction, which will be useful in the following to build the constrained system.

\subsection{Constraints Graph}

Constraints between normal vectors have to be determined on the reduced set of directions. These constraints are represented as arcs in a so-called "constraints graph" (see figure 1(e)]. In this unoriented graph, each direction is a node. Several types of valued arcs are added between two nodes $\mathbf{v}_{i}$ and $\mathbf{v}_{j}$ whenever a condition is met:

- orthogonality if $\left|\mathbf{v}_{i} \bullet \mathbf{v}_{j}\right|>\cos \left(\sigma_{\varangle}\right)$

- vertical symmetry if $\left|\left(\mathbf{v}_{i}+\mathbf{v}_{j}\right) \bullet \mathbf{z}\right|>\cos \left(\sigma_{\varangle}\right) \cdot\left\|\left(\mathbf{v}_{i}+\mathbf{v}_{j}\right)\right\|$

- horizontal line if $\left|\mathbf{v}_{i} \otimes \mathbf{v}_{j}\right|<\sin \left(\sigma_{\varangle}\right) \cdot\left\|\mathbf{v}_{i} \otimes \mathbf{v}_{j}\right\|$

where $\bullet$ represents the dot product and $\mathbf{z}$ is the vertical direction. Each arc $c$ corresponds to a constraint between two directions $\mathbf{v}_{i}$ and $\mathbf{v}_{j}$, which will be noted $c_{f}=\mathbf{v}_{i}, c_{t}=\mathbf{v}_{j}$. In the constraints graph, arc (or constraint) orientation has no importance (graph is unoriented). In the following however, the previous notation will state that $c$ is oriented from $\mathbf{v}_{i}$ to $\mathbf{v}_{j}$, thus that $\mathbf{v}_{i}$ constraints $\mathbf{v}_{j}$. Only one arc may exist between two nodes, therefore in case of redundancy between vertical symmetry and horizontal line, symmetry is preferred since it brings more constraints on directions. In the case of building reconstruction, orthogonality is only tested between vertical planes. Let us mention that, as $\sigma_{/ /}, \sigma_{\varangle}$ is easily choosen according to the error estimated in the approximate model or the degree of caricature desired in the reconstruction.

\subsection{Dependences Graph}

One of the difficulties of methods performing an implicit parameterization of the model [10,9] is that directions must be computed sequentially so that they can be deduced from some previous ones in the sequence and some constraint rules. This is equivalent to orient the constraints graph [10] while ensuring that no over-constraint occurs. Some attempts have been made to solve this complex problem in general configurations [13] but they are not well adapted to the 3D case. Another approach has been developed to handle general configurations (with constraints on points, planes, lines...) with procedures designed to maximize the number of constraints applied in the system [10]. We reuse some of the procedures to orient our constraint graph.

The algorithm explicitely builds the sequence of directions. Initially, all constraints in the constraints graph are considered inactive. The algorithm iteratively integrates all directions in the sequence and orients therefore the constraints (equivalent to arcs) of the constraints graph linked to them. Let us mention that each oriented arc reduces the 
degree of freedom of the node it gets to by $f(e): 2$ when it corresponds to vertical symmetry, 1 for any of the two other constraints. Initially, each node has a degree of freedom of 2 and the current degree of freedom can easily be computed at each step and for each node.

First the vertical direction (that has a peculiar significance in building reconstruction) is introduced in the sequence and all constraints linked to that node are made active and oriented from it. Then iteratively, the algorithm chooses in the most constrained directions one that has the highest number of potential (still non active) constraints related to it. It activates these constraints by orienting them from this node. If, at any point a direction gets over-constrained (its degree of freedom becomes negative), some constraints are suppressed until there is no more over-constraint.

At the end of this process, the "dependences graph" is the result of the orientation of the arcs in the constraints graph. Let us note that some constraints may disappear between the constraints graph and the dependences graph (see figure $1(\mathrm{e})$ ) due to some possible cases of overconstraints during the process or because of redundancy. Once the dependences graph is built, it can be shown that for each direction, only 6 situations recalled in table 1 may occur. From the previous procedure, each direction may also be numbered so that they can be computed sequentially, each one being defined by the previous ones and some constraints applied on it, which are given by arcs of the dependences graph. In the following, we will assume that the directions have been renumbered and that $\forall i, \mathbf{v}_{i}$ depend only on previous direction(s) $\mathbf{v}_{j}, j<i$. Whenever a direction $\mathbf{v}_{i}$ is not completely constrained by previous directions in the sequence (for instance arbitrary directions that do not depend on any previous direction in the sequence or perpendicular directions that depend on only one other direction), one may assume that it depends on some parameters $\boldsymbol{\theta}_{i}$. Some of the directions may not require any parameter since they are completely defined by other directions (or are known!). However, for simplicity, we will keep these useless $\boldsymbol{\theta}_{i}$ in our notation and consequently consider the set $\boldsymbol{\theta}_{1} \cdots \boldsymbol{\theta}_{D}$. Then rules recalled in the table 1 enable to sequentially compute each direction from these parameters and directions of dependences so that geometrical constraints imposed to them are verified. From this table, derivatives $\partial \mathbf{v}_{i} / \partial \boldsymbol{\theta}_{k} \quad \forall i, k$ can easily be deduced from given hints and chain rule. They will be of use in the iterative minimization.

\section{Enforcing Constraints}

\subsection{Geometrical Parametrization}

This section mostly recalls principles of the method depicted in [14] As explained above, each direction is computed from some parameters $\boldsymbol{\theta}=\left[\boldsymbol{\theta}_{1}^{T}, \ldots, \boldsymbol{\theta}_{D}^{T}\right]^{T}$. Each pair of points belong to the same plane whose normal direction is $\mathbf{v}_{i}$ if and only if their coordinates $\mathbf{X}_{m}, \mathbf{X}_{n}$ verify the planarity constraint:

$$
\mathbf{v}_{i}^{T}\left(\mathbf{X}_{m}-\mathbf{X}_{n}\right)=0
$$

For each facet of the initial model with $|f|$ points, $|f|-1$ equations can thus be inferred. Concatenating all equations leads to the system $\mathbf{B}\left(\boldsymbol{\theta}_{1}, \boldsymbol{\theta}_{2}, \ldots, \boldsymbol{\theta}_{D}\right) \mathbf{X}=\mathbb{O}$ where $\mathbf{X}=$ 
Table 1. Computations of direction $\mathbf{v}_{i}$ and its derivatives with respect to some parameters and according to relations constraining them. $\mathbf{S}_{\mathbf{v}}$ is the skew symmetric matrix for a vector $\mathbf{v}$

\begin{tabular}{|c|c|c|c|}
\hline Type & \multicolumn{2}{|c|}{ Parameter|Value } & Derivatives \\
\hline known & No & unchanged & $\mathrm{O}$ \\
\hline arbitrary & $\boldsymbol{\theta}_{i} \neq \mathrm{O}$ & $\mathbf{v}_{i}=\frac{\boldsymbol{\theta}_{i}}{\left\|\boldsymbol{\theta}_{i}\right\|}$ & $\frac{\partial \mathbf{v}_{i}}{\partial \boldsymbol{\theta}_{i}}=\frac{1}{\left\|\boldsymbol{\theta}_{i}\right\|}\left(I-\frac{1}{\left\|\boldsymbol{\theta}_{i}\right\|^{2}} \boldsymbol{\theta}_{i} \boldsymbol{\theta}_{i}^{T}\right)$ \\
\hline orthogonal to $\mathbf{v}_{j}$ & $\boldsymbol{\theta}_{i}$ & $\begin{array}{l}\mathbf{u}_{1}=\left(I-\mathbf{v}_{j} \mathbf{v}_{j}^{T}\right) \boldsymbol{\theta}_{i} \\
\mathbf{u}_{2}=\frac{\mathbf{u}_{1}}{\left\|\mathbf{u}_{1}\right\|}, \mathbf{v}_{i}=\mathbf{u}_{2}\end{array}$ & $\begin{array}{l}\frac{\partial \mathbf{v}_{i}}{\partial \boldsymbol{\theta}_{i}}=\frac{1}{\left\|\mathbf{u}_{1}\right\|}\left(I-\mathbf{u}_{2} \mathbf{u}_{2}^{T}-\mathbf{v}_{j} \mathbf{v}_{j}^{T}\right) \\
\frac{\partial \mathbf{v}_{i}}{\partial \mathbf{v}_{j}}=\frac{1}{\left\|\mathbf{u}_{1}\right\|}\left(\left(\mathbf{v}_{j}^{T} \boldsymbol{\theta}_{i}\right)\left(I-\mathbf{u}_{2} \mathbf{u}_{2}^{T}\right)+\mathbf{v}_{j} \boldsymbol{\theta}_{i}^{T}\right)\end{array}$ \\
\hline 2 orthogonalities: $\mathbf{v}_{j}, \mathbf{v}_{k}$ & No & $\mathbf{v}_{i}=\frac{\mathbf{v}_{j} \otimes \mathbf{v}_{k}}{\left\|\mathbf{v}_{j} \otimes \mathbf{v}_{k}\right\|}$ & $\begin{array}{l}\frac{\partial \mathbf{v}_{i}}{\partial \mathbf{v}_{j}}=-\frac{1}{\left\|\mathbf{v}_{j} \otimes \mathbf{v}_{k}\right\|}\left(I-\mathbf{v}_{i} \mathbf{v}_{i}^{T}\right) \mathbf{S}_{v_{k}} \\
\frac{\partial \mathbf{v}_{i}}{\partial \mathbf{v}_{k}}=\frac{1}{\left\|\mathbf{v}_{j} \otimes \mathbf{v}_{k}\right\|}\left(I-\mathbf{v}_{i} \mathbf{v}_{i}^{T}\right) \mathbf{S}_{v_{j}}\end{array}$ \\
\hline horizontal line with $\mathbf{v}_{j}$ & $\boldsymbol{\theta}_{i}$ & $\begin{array}{l}\mathbf{h}=\mathbf{z} \otimes \mathbf{v}_{j} \\
\mathbf{u}_{1}=\left(I-\mathbf{h h}^{T}\right) \boldsymbol{\theta}_{i} \\
\mathbf{u}_{2}=\frac{\mathbf{u}_{1}}{\left\|\mathbf{u}_{1}\right\|}, \mathbf{v}_{i}=\mathbf{u}_{2}\end{array}$ & $\begin{array}{l}\frac{\partial \mathbf{v}_{i}}{\partial \boldsymbol{\theta}_{i}}=\frac{1}{\left\|\mathbf{u}_{1}\right\|}\left(I-\mathbf{u}_{2} \mathbf{u}_{2}^{T}-\mathbf{h} \mathbf{h}^{T}\right) \\
\frac{\partial \mathbf{v}_{i}}{\partial \mathbf{v}_{j}}=\frac{1}{\left\|\mathbf{u}_{1}\right\|}\left(\left(\mathbf{h}^{T} \boldsymbol{\theta}_{i}\right)\left(I-\mathbf{u}_{2} \mathbf{u}_{2}^{T}\right)+\mathbf{h} \boldsymbol{\theta}_{i}^{T}\right) \mathbf{S}_{\mathbf{z}}\end{array}$ \\
\hline vertical symmetry with $\mathbf{v}_{j}$ & & $\mathbf{v}_{i}=2\left(\mathbf{v}_{j} \cdot \mathbf{z}\right)-\mathbf{v}_{j}$ & $\frac{\partial \mathbf{v}_{i}}{\partial \mathbf{v}_{j}}=2 \mathbf{z z}^{T}-I$ \\
\hline
\end{tabular}

$\left[\mathbf{X}_{1}^{T}, \ldots, \mathbf{X}_{N}^{T}\right]^{T}$ holds all the points coordinates and $\mathbf{B}\left(\boldsymbol{\theta}_{1}, \boldsymbol{\theta}_{2}, \ldots, \boldsymbol{\theta}_{D}\right)$, a $P \times 3 N$ matrix, holds the geometrical constraints. It is easy to see that the dimension $M$ of the nullspace of $\mathbf{B}$ is greater than 3 and thus an implicit parameterization of the points $\mathbf{X}$ is

$$
\mathbf{X}=\mathbf{U}\left(\boldsymbol{\theta}_{1}, \boldsymbol{\theta}_{2}, \ldots, \boldsymbol{\theta}_{D}\right) \mathbf{V}
$$

with $\mathbf{V} \in \mathbb{R}^{M}$ and where $\mathbf{U}\left(\boldsymbol{\theta}_{1}, \boldsymbol{\theta}_{2}, \ldots, \boldsymbol{\theta}_{D}\right)$ ( $\mathbf{U}$ for brevity) is a $3 N \times M$ matrix whose columns form an orthonormal basis of the nullspace of $\mathbf{B}$. Thus $\mathbf{X}$ implicitly verify all the geometrical constraints and $\mathbf{V}$ holds the implicit parameters of the shape. By sequential construction of the dependences graph, normal vectors verify the geometrical constraints and thus any values for $\mathbf{V}$ supply a solution for the constrained system. Consequently, $M$ is the number of degrees of freedom of $\mathbf{X}$.

As for the unknowns, $\boldsymbol{\theta}$ and $\mathbf{V}$, they are collected in a single vector $\boldsymbol{\Theta}=[\boldsymbol{\theta}, \mathbf{V}]$.

\subsection{Maximum Likelihood Reconstruction}

We choose to estimate the solution subject to geometrical constraints that is most likely to verify the initial 3D observations. The solution should then minimize the function:

$$
\mathcal{Q}(\boldsymbol{\Theta})=\left\|\mathbf{X}_{i n i}-\mathbf{X}(\boldsymbol{\Theta})\right\|^{2}
$$

A classical iterative Levenberg-Marquardt procedure is performed for this minimization. We assume the reader is familiar with Levenberg-Marquardt algorithm and highlight some of the important difficulties related to the computations of the cost function and its derivatives at different steps $i$. The initialization of $\boldsymbol{\theta}_{i}$ is directly linked to the initial values of the normal vectors of each facet. As for $\mathbf{V}$, we choose $\mathbf{V}^{0}=$ $\left(\mathbf{U}^{0}\right)^{+} \mathbf{X}_{i n i}$ where $\left(\mathbf{U}^{0}\right)^{+}$stands for the pseudo-inverse of $\mathbf{U}^{0}$. $\mathbf{U}^{0}$ can be chosen arbitrarily as orthonormal basis of the nullspace of $\mathbf{B}$ but some provisions must then be taken to ensure the unicity of $\mathbf{U}$ in the next steps of the iterative process. Starting from an initial value $\mathbf{U}^{s}$, and assuming $\mathcal{U}_{1}$ is any unitary matrix whose columns 
form a basis of $\mathcal{N} u l l\left(\mathbf{B}\left(\boldsymbol{\theta}^{s}\right)\right)$, the procedure 4 ensures that $\mathbf{U}^{i}$ is uniquely defined.

$$
\begin{array}{r}
\mathbf{U}_{\mathbf{1}}{ }^{T} \mathbf{U}^{s} \stackrel{\mathrm{SVD}}{=} \mathcal{U} \mathcal{D} \mathcal{V}^{T} \\
\mathbf{U}^{i}=\mathbf{U}_{1} \mathcal{U} \mathcal{V}^{T}
\end{array}
$$

It can also be shown that, thanks to this procedure, $\mathbf{U}^{i}$ is differentiable in a neighborhood of $\boldsymbol{\theta}^{s}$ and derivatives are given by 5 where $\mathbf{B}^{+}$stands for the pseudo-inverse of $\mathbf{B}$ and $\frac{\partial \mathbf{B}}{\partial \boldsymbol{\theta}}\left(\boldsymbol{\theta}^{s}\right)$ is trivial to compute. From these relations, evaluation and derivatives of $\mathcal{Q}\left(\boldsymbol{\Theta}^{i}\right)$ become straightforward.

$$
\frac{\partial \mathbf{U}}{\partial \boldsymbol{\theta}}\left(\boldsymbol{\theta}^{s}\right)=-\mathbf{B}^{+} \frac{\partial \mathbf{B}}{\partial \boldsymbol{\theta}}\left(\boldsymbol{\theta}^{s}\right) \mathbf{U}^{s}
$$

\section{Handling Over-Constraints and Degeneracies}

\subsection{Over-Constraints and Degeneracies Detection}

The dependences graph generation ensures that no over-constraint on directions can occur. However, in the constrained system applied to points, nothing ensures that a point is not over-constrained. This remark comes from the fact that the minimization is done in two steps as in [1]: first, directions are computed, and then points must conform to these directions. It may be possible that points can not verify all planarity relations because, for instance, in the directions computation, no test has been performed to verify some situations that topology may impose. Degeneracies occur when values are too far away from the constrained situation imposed by the application. In this case, unable to find a acceptable solution, implicit parameterization merge two points and supplies a degenerate solution.

In our scheme, over-constrained situations as well as degeneracy situations are easily detected, as stated in [14] by examining $\mathbf{U}^{i}$ at any step of the algorithm. If any triplets $\{3 m-2,3 m-1,3 m\}$ and $\{3 n-2,3 n-1,3 n\}(m \neq n)$ of rows, corresponding to two different points are equal, it means that the only available solution was to merge these two points and a degeneracy has been detected.

\subsection{Over-Constraints and Degeneracies Handling}

A good way of dealing with this would be an automatic property detection that could add some additional constraints to the directions set due to topology considerations [10]. In our case, when degeneracies or overconstraints are detected during geometric parametrization, constraints are iteratively removed until no more degeneracies or overconstraints occur, and the minimization process is reinitialized. The heuristic algorithm uses procedures to favor constraints whose suppressing decrease the least the global number of degrees of freedom in order to keep as many constraints as possible. At the end of the algorithm, in case of success, all topological relations are verified insofar as all planarity constraints on facets embedded in the implicit parameterization are kept. 


\section{Results}

\subsection{General Protocol}

For simulation purpose, 25 reference buildings have been obtained from automatic algorithm [2] (without constraints), corresponding to 238 points. From this modelling, constraints are inferred and enforced using the algorithm depicted, thus giving a constrained reference that will be the basis of our comparison: all results will be made with regard to this (constrained) reference. To evaluate our algorithm, gaussian noise is added to points with an increasing standard deviation and reconstruction is performed, taking these noisy points as $\mathbf{X}_{i n i}$ in the algorithm. For all results given, average is made on 25 iterations. parameters used were: $\sigma_{/ /}=12^{\circ}$ and $\sigma_{\varangle}=10^{\circ}$.

Two cases are considered for evaluation on noisy buildings: a priori constraints and a posteriori constraints. The first case is comparable to that of model-based reconstruction: constraints are known a priori by an external information (brought by constraints inferred from the reference models), thus avoiding the directions clustering and constraint graph building step (but initial values are obviously computed from the noisy buildings). In the second case, constraints are systematically inferred from the noisy model. It is therefore obvious that some constraints will be lost compared to the reference buildings.

On the one hand, we analyze the gain in precision, that is the average distance between reconstructed buildings and constrained reference. Recall that minimization is done however with regard to the noisy points ! On the other hand gain in degree of freedom is studied to show the rigidity that constraints bring to reconstruction. Without any constraint, the global degree of freedom of the 25 modelled buildings is 966 .

\subsection{A priori}

In this case, as can be seen for some noise in figure 2, reconstruction is close to what is expected, the global shape is much more acceptable. Figure 3 shows more quantitative results. One can see that distance is always lower than without constraint, which states that constraints naturally tend to get closer to reference. In the case of a priori constraints, reduction of degree of freedom is only due to over-constraints or degeneracies detected in the minimization process and that led to constraints suppression. A great stability to noise can be seen for a priori constraint that is not sensitive to noise until $\sigma=0.5 \mathrm{~m}$. Let us note also that a manual evaluation of degree of freedom of the buildings has showed that 258 was the expected degree of freedom, which is the number found by the algorithm when noise is low. It proves thus that no constraints is lost during the process and particularly during the dependence graph construction step on the reference and therefore validates our procedures.

\subsection{A posteriori}

Although all the constraints are not inferred as soon as noise increases, global shape of buildings is still much more acceptable than without constraints (Figure 2). Let us note that $\sigma=0.3$ is an important noise especially when one-meter edges are present in the 

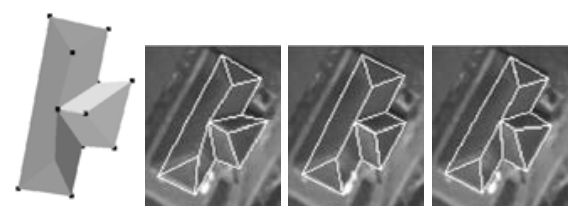

(a) $48 / 10 / 11$
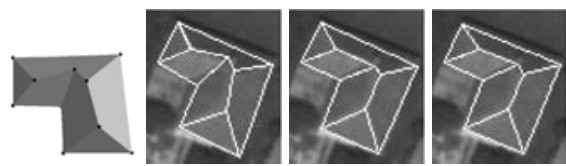

(b) $42 / 10 / 10$

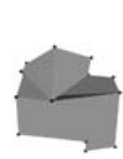

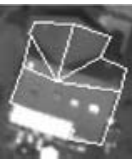

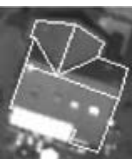

(c) $54 / 14 / 15$
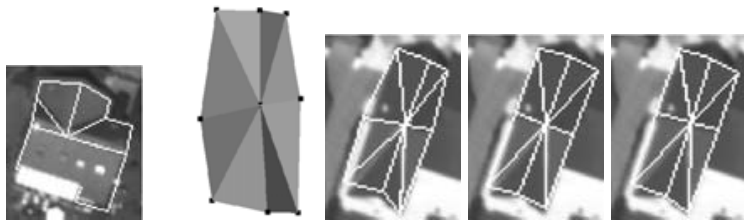

(d) $39 / 7 / 12$

Fig. 2. Some views of several buildings with $\sigma=0.5 \mathrm{~m}$. first column: views of noisy buildings in 3D. 3 next columns: views of a noisy builing, a priori constraints enforcing and a posteriori constraints enforcing. The three numbers indicates the number of degrees of freedom for reconstruction without constraint, with a priori constraints and with a posteriori constraints respectively
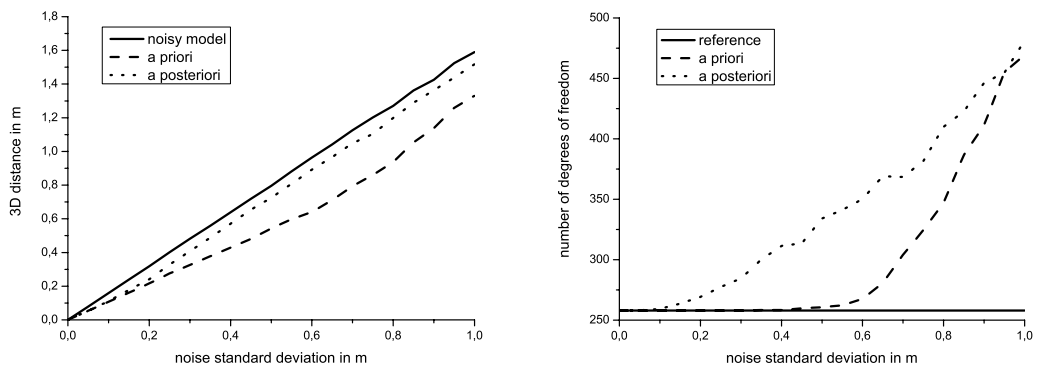

Fig. 3. left: distance to the reference. right: number of degrees of freedom. In each graph, $\mathrm{x}$-axis corresponds to standard deviation of noise added on points

scene. As far as degree of freedom is concerned, a relative stability can be observed up to $\sigma=0.3$ where only $10 \%$ of degrees of freedom is lost.

\section{Summary and Conclusion}

We have presented a method for automatic inferring and enforcing constraints on 3D models. This method requires no user intervention for constraints definition and, by implicitly parameterizing the model, enables to know exactly which constraints are verified on the reconstructed model. The approach uses few thresholds and han- 
dles over-constraints by iteratively removing some constraints. Results show the gain obtained by this algorithm as far as degree of freedom and precision are concerned.

Future work include the integration of image features in the whole loop so as to fit the constrained models on points of interest in the images. This would solve some of the artefacts visible in 2 where edges of the constrained model do not match perfectly segments of images. Fitting a 3D model on image features is a difficult problem since mismatched and non-matched features must be handled. In this case, the model should take full benefit of the reduction of degree of freedom due to constraints to overcome nonmatched or mismatched features. Another point to study is the possibility of overcoming over-constraint without heuristic suppressing of constraints. Automatic property detection seems promising to add implicit constraints due, for instance, to topology.

\section{References}

1. Shum, H.Y., Han, M., Szeliski, R.: Interactive construction of 3d models from panoramic mosaics. In: Conference on Computer Vision and Pattern Recognition. (1998) 427-433

2. Taillandier, F., Deriche, R.: Automatic buildings reconstruction from aerial images: a generic bayesian framework. In: Proceedings of the XXth ISPRS Congress, Istanbul, Turkey (2004)

3. Debevec, P., Taylor, C., Malik, J.: Modeling and rendering architecture from photographs: a hybrid geometry-and image-based approach. In: SIGGRAPH 96. (1996) 11-20

4. Suveg, I., Vosselman, G.: 3D reconstruction of building models. In: Proceedings of the XIXth ISPRS Congress. Volume 33., Amsterdam (2000) B3:538-545

5. Fischer, A., Kolbe, T., Lang, F., Cremers, A., Förstner, W., Plümer, L., Steinhage, V.: Extracting buildings from aerial images using hierarchical aggregation in $2 \mathrm{D}$ and $3 \mathrm{D}$. Computer Vision and Image Understanding 72 (1998) 163-185

6. Jaynes, C., Riseman, E., Hanson, A.: Recognition and reconstruction of buldings from multiple aerial images. Computer Vision and Image Understanding 90 (2003) 68-98

7. Noronha, S., Nevatia, R.: Detection and modeling of buildings from multiple aerial images. IEEE Transactions on Pattern Analysis and Machine Intelligence 23 (2001) 501-518

8. Kim, Z., Huertas, A., Nevatia, R.: Automatic description of buildings with complex rooftops from multiple images. In: Conference on Computer Vision and Pattern Recognition. Volume 2. (2001) 272-279

9. Grossmann, E., Santos-Victor, J.: Maximum likelihood 3d reconstruction from one or more images under geometric constraints. In: British Machine Vision Conference. (2002)

10. Bondyfalat, D.: Interaction entre Symbolique et Numérique; Application à la Vision Artificielle. Thèse de doctorat, Univ de Nice Sophia-Antipolis (2000)

11. van den Heuvel, F.A.: A line-photogrammetric mathematical model for the reconstruction of polyhedral objects. In: Videometrics VI, SPIE. Volume 3641. (1999) 60-71

12. Ameri, B., Fritsch, D.: Automatic 3D building reconstruction using plane-roof structures. In: ASPRS, Washington DC (2000)

13. Hoffmann, C., Vermeer, P.: Geometric constraint solving in $R^{2}$ and $R^{3}$. In: Computing in Euclidean Geometry. $2^{\text {nd }}$ edn. World Scientific Publishing (1995) 266-298

14. Grossmann, E.: Maximum Likelihood 3D Reconstruction From One or More Uncalibrated Views Under Geometric Constraints. PhD thesis, Universidade Tecnica de Lisboa (2002) 